\title{
Supporting Smart Electric Vehicle Charging with Information-Centric Networking
}

\author{
Konstantinos V. Katsaros*, Wei Koong Chai*, Barbara Vieira ${ }^{\dagger}$ and George Pavlou* \\ *University College London, UK \\ E-mail: \{k.katsaros, w.chai, g.pavlou\}@ucl.ac.uk \\ ${ }^{\dagger}$ Radboud University Nijmegen, NL \\ E-mail: b.vieira@cs.ru.nl
}

\begin{abstract}
Inspired by the proliferation of content-centric applications in the Internet, Information-Centric Networking (ICN) has emerged as a promising networking paradigm. Focusing on the delivery of content instead of the pairwise communication between end-hosts, ICN inherently supports locationindependent content/information distribution, through the means of in-network caching and multicast; as well as mobile computing. However, so far the vast majority of ICN research efforts have mostly focused on the design of sound and scalable architectures and protocols for the current Internet application landscape. In this paper, we revisit ICN in the context of a radically different application environment of smart grids and in particular, the case of smart charging of electric vehicles. Based on a thorough description of the currently forming application environment in the Netherlands, we highlight the inefficiencies resulting from a host-centric model. We then show how ICN can address these limitations and ultimately support quality and security in such application environment. Besides qualitative benefits, our preliminary analysis also demonstrates that ICN can substantially reduce communication and security complexity, thus fostering the development and widespread adoption of the smart charging application.
\end{abstract}

Index Terms - smart grid, electric vehicles, caching, in-network processing, congestion management, load balancing

\section{INTRODUCTION}

Information-centric networking (ICN) has attracted significant interest in the research community during the last years. Supporting routing by name, ICN achieves locationindependence and enables a series of valuable features such as in-network caching, multicast and mobility support [1]. Such features are particularly desirable for applications related to the delivery of content to both fixed and mobile users. Indeed following efforts on peer-to-peer content distribution [2] and content delivery networks (CDNs) [3], research on ICN has been largely motivated by the proliferation of content-centric applications in the Internet. In addition to that, by binding names to the content / information itself, ICN also allows inherent security features rather than relying on a posteriori patches.

While most ICN works relate to Internet applications, recent works have started to advocate the adoption of the ICN networking paradigm in addressing security and resilience concerns in the context of smart grids [4], mostly focusing on power grid monitoring and home energy management applications [5]-[7]. In this work, we argue that the ICN paradigm can be further leveraged to support and facilitate communications in the emerging smart electric vehicle charging applications [8]-[10]. Following advances in several key technologies such as power electronics, energy storage and embedded software, reliable electric vehicles are starting to penetrate the transportation landscape. With sustainability in mind, some governments are already providing incentives for the use of the more environmentally friendly electric vehicles. For instance, the United Kingdom government offers a Plug-in Car Grant ${ }^{1}$ of up to $£ 5000$ while the U.S. federal government provides tax credits up to $\$ 7500^{2}$ to electric vehicle buyers.

In this context, the grid-to-vehicle $(\mathrm{G} 2 \mathrm{~V})$ charging application environment has started shaping, where a series of different autonomous, administrative entities/actors such as electric vehicles (EVs), distribution system operators (DSOs), energy providers (EPs), charging station operators (CSOs), need to share and exchange information in a complex application environment. Often the same information must be exchanged by multiple entities at different time-scales and granularities, resulting in a complicated set of information flows. In addition, information may require different levels of security and privacy clearances (e.g., for charging and billing purposes). Moreover, communications in this domain also impact critical operations related to the stability of the power grid.

In this paper, we show how the spatiotemporal decoupling of communicating entities in ICN, and the support for in-network caching, multicast and mobility, can be leveraged to support the increasingly demanding communication environment of smart charging applications. Inherently enabling locationindependence, ICN can substantially reduce the complexity of establishing an entire mesh of point-to-point communication sessions in a host-centric networking context, which in turn can result in an error prone, unreliable and unscalable communication environment. Moreover, by securing the information itself, rather than the multitude of pairwise communication channels, ICN can further support the security requirements of the smart charging application domain. Finally, we show

\footnotetext{
${ }^{1}$ https://www.gov.uk/government/publications/plug-in-car-grant

${ }^{2} \mathrm{http}: / /$ www.afdc.energy.gov/laws/law/US/409
} 
how lightweight in-network processing, enabled by namebased routing in ICN can be employed to further reduce communication complexity and network traffic, but also the CAPEX for the realization of smart charging.

In the following, we first provide a thorough description of the smart charging application environment. Building on input from a real use case in the Netherlands, we present a generic application model with a particular focus on the involved communicating entities, their relationships, the resulting information flows and the related security requirements (Section II). Then, in Section III, we elaborate on how ICN can support quality, reliability and security of smart charging applications. Based on a preliminary analysis of the application environment, we quantify the benefits brought by ICN in Section IV, and finally conclude in Section V.

\section{SMART CHARGING COMMUNICATION ENVIRONMENT}

The electrification of the transportation system poses significant challenges to the existing power grid infrastructure [11]. The steadily increasing penetration of EVs substantially raises the load on the power grid. For instance, as reported in [12], an average Dutch household uses $3600 \mathrm{kWh} /$ year, while an electric vehicle will need approximately 3000-4000 $\mathrm{kWh} /$ year. What is more, the power demand of EVs presents strong spatiotemporal patterns with a significant fraction of the EV population charging at home during the night or at work. At the same time, the expected increase of EV penetration (see [13] and references therein) is also expected to result in energy demand while on the move, i.e., using public charging stations while traveling, where the demand can significantly fluctuate.

In this general environment, a series of challenges emerge not only for the DSOs but also for other actors emerging in the realization of the so-called smart charging application. Based on the smart charging model currently being shaped in the Netherlands, we introduce in the following the actors involved and describe their particular roles. We pay particular attention to the relationships established amongst the different actors as these largely affect the resulting communication requirements. Based on these, we then present a baseline application scenario for the ensuing discussion.

\section{A. Actors, roles and relationships}

1) Distribution System Operators (DSOs). The entity that operates the power distribution grid. It distributes energy generated by energy providers to energy consumers, including the electric vehicles, over its power grid infrastructure. Since a common infrastructure is used for the delivery of energy to other types of consumers (e.g., households), the DSO is responsible for estimating the residual capacity of the power cables to be used for smart charging.

2) Energy providers (EPs). Autonomous entities producing energy. Traditionally, this refers to power stations (e.g., coal, fossil-fuel, nuclear) which deliver high-voltage energy to the power grid. With the advent of smart grid, other EPs known as distributed renewable energy resources (DRERs) such as wind/solar farms have emerged, providing renewable but more volatile energy.

3) Electric vehicles (EVs). Here we consider plug-in EVs. Each EV is under the autonomous control of its owner. The charging requirements and preferences of the owner (e.g., preferred charging times, mobility patterns, charging cost thresholds) comprise the charging profile of the EV.

4) E-Mobility Service Providers (EMSPs). The entities providing the charging service to EV owners. They are responsible for managing customer information and billing. EMSPs obviously provide their services to multiple EVs; though an EV is typically expected to have an agreement with a single EMSP.

5) Charging Stations (CSs). The stations where EVs can plug-in and recharge their battery. Currently, CSs are equipped with two sets of metering devices: (i) a metering device at each charging spot of the CS, measuring the individual consumption of each EV; this is used for billing and profiling purposes (see next), and (ii) a metering device measuring the overall energy consumption of the entire CS; this is owned and managed by the DSO and is used to get the aggregate consumption information required by the EP to charge the CSO. The common practice currently is for the two metering devices to be equipped with separate wireless network interfaces connecting them to the CSO and DSO respectively.

6) Charging Station Operator (CSOs). Entities responsible for the operation and maintenance of CSs. A CSO usually manages multiple CSs. A CSO can be an autonomous actor or an off-shoot of an EMSP. In the former case, a CSO may establish relationships with multiple EMSPs so as to provide their services to a wider set of EVs. At the same time, EMSPs establish agreements with more than one CSO to allow their customers charge their cars in an increased number of CSs. Each CSO has a contract with a single EP, though more complicated scenarios are envisioned for the future where CSOs establish relationships with multiple smallfootprint EPs.

7) Energy Data Service Netherland (EDSN). Platform for administrative connections between various actors within the energy sector, providing a common interface to exchange metering data. EDSN has been set up by $\mathrm{NEDU}^{3}$, a collective of DSOs, being for that reason the most reliable/trustworthy entity in the electricity chain of the Netherlands. In essence, EDSN represents a commonly trusted authority; as such, it can also be envisioned to be realised by the regulation authorities of a country.

It becomes evident that smart charging involves a wide set of actors with a variety of one-to-one, one-to-many and manyto-many relationships amongst them. Figure 1 illustrates these

\footnotetext{
${ }^{3}$ NEDU stands for Vereniging Nederlandse Energie Data Uitwisseling.
} 
relationships. As it will become evident by the description of a typical application scenario (see next), these relationships result in a complex set of communication flows between the involved actors.

\section{B. Baseline application scenario}

The baseline functionalities of smart charging applications include billing and congestion management. In the former case, communication amongst the various actors takes place so that energy consumers pay their providers. Specifically, from the relationships depicted in Figure 1, EV owners are billed by EMSPs (a) and EMSPs are billed by CSOs (b), which in turn are billed by EPs (c). When plugging-in, EV owners denote their identity for billing purposes (d), usually through a smart card. The CSO receives this information from the CS (e) and queries the EMSP to check the validity of the card identifier (f).

A metering device at the CS then measures the amount of energy provided to each EV so that the owner is billed accordingly. To this end, each metering value is delivered from the CS to the CSO (g), and from there to the EMSP (h). At the same time, the DSO metering device at each CS is responsible for providing the aggregate consumption measurements for the billing of CSOs by EPs. These measurements are delivered from each CS directly to the DSO (i) and then become available to EPs through the EDSN (j).

The purpose of congestion management in this baseline scenario is to ensure the availability of the required power at the CSs without exceeding the maximum capacity of the cables. This is because the aggregate EV demand may well exceed the capacity of the cables ${ }^{4}$. Statically capping the available energy supply at each CS results in a sub-optimal usage of the network as demand typically varies both in time and space. Hence, congestion management aims at adapting the allocation of power to each CS. To this end, DSOs estimate the residual capacity of their cables based on historical consumption data of households and other energy consumers (e.g., industrial sites), and weather forecasts. These estimations are then made available to the CSOs (k) and subsequently to the EMSPs (l). The CSOs decide how to distribute the available energy to the incoming EVs at a CS level.

On the EV side, users autonomously select the CS to charge. These decisions may be based on recommendations targeting the balance of load in the power grid, the minimization of waiting time, etc. [8]-[10]. Based on their profiles, EVs can have different charging behaviours, e.g., charging at a lower rate for a lower price instead of the full charging rate. Table I summarizes the basic information exchanges taking place in this baseline scenario. Figure 1 provides an overview of the smart charging environment.

\section{ICN FEATURES AND BENEFITS}

A closer look at the described application environment reveals that following the host-centric networking paradigm

\footnotetext{
${ }^{4}$ Note that congestion in this case refers to the power grid operations and not the communications domain.
}

TABLE I

INFORMATION FLOWS IN THE BASELINE SMART CHARGING SCENARIO.

\begin{tabular}{|c|c|c|c|}
\hline Rel & Src Actor & Information & Dst Actor \\
\hline $\mathrm{a}$ & EMSP & Billing data & EV \\
\hline $\mathrm{b}$ & $\mathrm{CSO}$ & Billing data & EMSP \\
\hline $\mathrm{c}$ & $\mathrm{EP}$ & Billing data & $\mathrm{CSO}$ \\
\hline $\mathrm{d}$ & $\mathrm{EV}$ & User identification data & $\mathrm{CS}$ \\
\hline $\mathrm{e}$ & $\mathrm{CS}$ & User identification data & $\mathrm{CSO}$ \\
\hline $\mathrm{f}$ & $\mathrm{CSO}$ & User identification data & EMSP \\
\hline $\mathrm{g}$ & $\mathrm{CS}$ & Meter readings, per EV & $\mathrm{CSO}$ \\
\hline $\mathrm{h}$ & $\mathrm{CSO}$ & Meter readings, per EV & EMSP \\
\hline $\mathrm{i}$ & $\mathrm{CS}$ & Aggregate DSO meter readings & DSO \\
\hline $\mathrm{j}$ & $\mathrm{DSO}$ & Aggregate DSO meter readings (through EDSN) & EP \\
\hline $\mathrm{k}$ & $\mathrm{DSO}$ & 24-hour forecast, 15 min granularity & $\mathrm{CSO}$ \\
\hline $\mathrm{l}$ & $\mathrm{CSO}$ & 24-hour forecast, 15 min granularity & EMSP \\
\hline
\end{tabular}

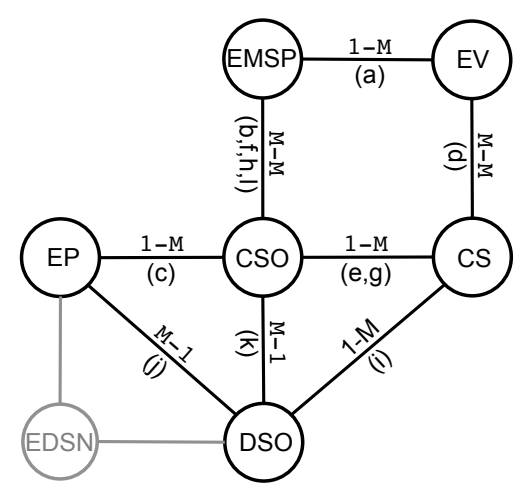

Fig. 1. Actors, relationships and information flows in smart charging. Edges denote the relationships between the actors (nodes) and are annotated with the business relationship type, i.e., one-to-many ( $1-\mathrm{M})$, many-to-one $(\mathrm{M}-1)$, many-to-many $(\mathrm{M}-\mathrm{M})$, as well as the information flow (see Table I).

can lead to a series of inefficiencies and limitations. We identify these in the following and show how they can be overcome by specific features enabled by ICN.

\section{A. Spatiotemporal decoupling}

From Table I, the existing smart grid scenario foresees the exchange of energy consumption measurements by several actors. Meter readings take place at the CS and the measurements are then eventually delivered (at different granularities) to CSO, EMSP, DSO and EP. Employing host-centric communication primitives in this context ${ }^{5}$ results in significant complexity and redundancy. While the aforementioned actors are interested in the same information, a series of point-topoint communication sessions needs to be established. This has two undesired effects. Firstly, the communication complexity increases severely with the increase of the number of actors in the system. As a result, management and control of the overall communication environment becomes cumbersome and prone to misconfiguration and errors. In consequence, the reliability and scalability of the system are questioned. Secondly, with the increase of EV penetration, data rates may significantly

\footnotetext{
${ }^{5}$ The Open Charge Point Protocol (OCPP) is an example of a host-centric protocol. It is one of the protocols most widely adopted, especially in Europe and Asia, yet it is currently not recognized by any national or international standards body. The situation in U.S. is more fragmented with proprietary protocols being used. However, in 2013, U.S. Department of Energy has made charge station network interoperability a priority.
} 
increase, challenging the low bandwidth transmission technologies currently used by operators (e.g., GPRS). This may have a significant impact on the quality of communications in the smart charging environment in the near future.

It is important to note that the nature of the relationships between the involved actors, and the corresponding business models, are expected to further amplify the complexity in the smart charging landscape. As shown in Figure 1, each actor may have multiple counterparts in its involvement in the smart charging application. For instance, several individual EMSPs may be interested in the meter readings of a wide set of CSs, while a multitude of small footprint EPs (e.g., DRERs) may also require access to such data. As a result, the number of pairwise communications in this environment is expected to increase, resulting in significant communication management overheads for the involved actors.

With ICN focusing on information rather than end-points, the communicating entities no longer require to establish a mesh of point-to-point communication sessions. In ICN, all the aforementioned entities can simply denote their interest in the energy consumption measurements and the network can efficiently cache / replicate the data when and where needed. For instance, both an EP and an EMSP may issue a request for the consumption measurements of the EMSP's clients at all involved CSs. The EP and EMSP, in this case, would not need to be aware of the network locations of all individual CSs and the resulting data would be replicated (or possibly cached) at the first common ancestor node of the EP and EMSP end hosts in the network, reducing duplicate traffic. It is important to note that by simply publishing or subscribing to the required data $^{6}$, new entities (e.g., a new CS) can also seamlessly enter the smart charging market without the need for complicated configurations (e.g., IP addressed, ports and credentials for each involved EP). In this way, an ICN architecture would enhance the scalability of the system, further facilitating the growth of the smart charging market.

\section{B. In-network processing and caching}

As briefly elucidated, individual entities in the network may be interested in the same information but in different granularity and/or scope. An EMSP is interested in finegrained individual meter readings to charge its customers, while an EP is only interested in the aggregate consumption, i.e., over all EVs charging in CSs of a certain CSO.

At the same time, a CS may report meter readings for EVs associated with different EMSPs and each EMSP should only receive the correct set of measurements. Accommodating such needs with the current host-centric networking paradigm requires the aforementioned aggregation and/or filtering of the reported values to take place at the edge of the network. In turn, this necessitates again the establishment of individual

\footnotetext{
${ }^{6}$ The publish and subscribe operations are considered inherent in the ICN paradigm, regardless of their exact realization. For instance, the PURSUIT architecture [14] is explicitly based on Pub/Sub primitives, while CCN [15] implicitly supports subscriptions and publications with Interest and Data messages, with any node being able to act as a broker entity [16].
}

point-to-point communication sessions for the delivery of the individual information flows, leading to the problems discussed in Section III-A.

We believe that following the ICN approach further opens the way for introducing new functionalities inside the network to address this limitation. Namely, by acting on named-data, an ICN network is in the position not only to inherently support in-network caching (see also Section III-D), but to further easily integrate primitive, light-weight processing functionalities such as information aggregation and filtering/scoping. Specifically, aggregation would act on the reported values in the payload of the messages, while filtering/scoping could be supported by including the appropriate information upon which the filtering/scoping criteria is applied in either content names or attributes of the content [17]. For instance, the name used to identify and forward metering readings can include the identifier of the involved EMSP.

Unlike in-network caching where the debate on whether ubiquitous or selective caches is better still rages (e.g., [18]), such in-network processing functions would be expected to be supported only at carefully selected locations in the network, so as to maximize effectiveness while reducing the computational resource requirements across the network, and the corresponding CAPEX and OPEX. This network planning would have to take into account the locations of the data, consumers and producers, the topological characteristics of the network and the characteristics of the information flows, e.g., data rates. Such research is beyond the scope of this paper. By supporting light-weight in-network processing of named data, an ICN network can further reduce the complexity of communications, again, enhancing scalability and thus the growth of the smart charging ecosystem. Note, however, that this does not violate the location-independence feature of ICN as the users are still not required to know the exact locations of each other.

\section{Security}

An inherent security advantage of ICN is that entities involved in the communication, i.e., senders and receivers of data, need not know each other's IP addresses. This spatiotemporal decoupling of senders and receivers reduces the risk of network-born attacks. This is especially relevant when some of the actors involved in the communication are often machines with limited capabilities, as it is the case, for instance, of the meters of the DSO installed at the CSs.

In the aforementioned scenario, many different actors are interested in the same type of information, requiring establishment of a considerable number of point-to-point connections. Most likely, such connections will rely on standard protocols (e.g., SSL/TLS or IPSec) to enforce the confidentiality and integrity of the information exchanged between the end parties. However, such protocols aim at securing the communication channel rather than the content itself. Trust in the content is intrinsically tied to the trust in the host the information comes from and how the content was retrieved. Therefore, EMPSs, for instance, will always have to trust that the EV meter 
readings produced by the CSs are not deliberately modified by the CSOs. Since ICN security models are focused on securing the content itself, adoption of ICN in this setting solves then some of these trust issues.

Even with respect to EV meter readings in the current setup, where enforcement of end-to-end security is required, it is important to highlight that ICN is indeed an asset. Observe that we can take advantage of the in-network functionalities supported by ICN to deploy privacy-friendly aggregation and filtering mechanisms by implementing security techniques based, for instance, on homomorphic encryption [19], [20] and public-key encryption with keyword search [21]. Note that smart grids in general have to obey the European regulations ${ }^{7}$ on data protection, so implementation of privacy-aware mechanisms to protect customers data is of utmost importance.

\section{Mobility support}

In the baseline scenario, EVs only communicate with a CS when they are plugged in, and with their EMSP via off-line means for billing. However, considering the already available communication capabilities of vehicles, EVs are expected in the future to also communicate while on the move. Going beyond the baseline scenario, recent works have started investigating scenarios in which EVs report their state of charge (SoC) while moving. Such information can prove valuable for congestion management, load balancing/scheduling purposes, e.g., EMSPs and CSOs can use SoC reports to accurately estimate the expected load and provide suggestions to the users so as to minimize their waiting time [8]. In other works, demand-response mechanisms are utilized to maximize green energy consumption [10].

The spatiotemporal decoupling of communicating entities in ICN is expected to prove beneficial in this context as well. Moving EVs can report their $\mathrm{SoC}$ when connected to the wireless network, cellular or through Road Side Units (RSUs). In an ICN environment, EVs do not need to establish a connection to each anticipated recipient, e.g., CSO/CS, EMSP. Note that this set of recipients is expected to be large in the future (e.g., there is already 492 CSs available in a 20 $\mathrm{km}$ radius of central London ${ }^{8}$ ), while mobility would also require frequent updates of each delivered information. At the same time, the increased penetration of EVs is expected to render centralized, possibly cloud-based, solutions impractical in terms of scalability and quality of experience.

On the downstream, EVs may receive pricing information without establishing an end-to-end communication session with their EMSP(s). In this scenario, in-network caching is also expected to prove beneficial effectively, supporting asynchronous communication. For instance, EVs may receive the latest pricing information from a cache located on the wireless base station/RSU.

\footnotetext{
${ }^{7}$ Directive $95 / 46 / \mathrm{EC}$ of the European Parliament on data protection: http://eur-lex.europa.eu/LexUriServ/LexUriServ.do?uri=CELEX: 31995L0046:en:HTML

${ }^{8}$ Information obtained from http://chargemap.com/ in July 2014.
}

\section{Preliminary AnAlysis}

In order to further illustrate the benefits of an ICN approach in reducing the communication complexity in the smart charging environment, we proceed with a simple comparative analysis of the communication requirements in the case of a plain IP and an ICN networking context. For simplicity, we focus here on the communication for the collection of the required metering information. We denote the number of involved EMSPs, EPs, DSOs, CSOs and CSs as $E, P, D$, $O$ and $S$, respectively. We also denote as $I$ the number of network nodes supporting ICN functionality in the network, i.e., name-based forwarding and in-network processing, as described above. Figure 2 provides a schematic illustration of the established information flows, with arrows showing the direction of data. For EMSPs, EPs, CSOs and CSs, we assume that one network node/host is employed by each actor in the system. However, in the case of DSOs, their large scale and geographical coverage would demand a noncentralised solution, as opposed to the current centralised SCADA deployments, with more than one nodes handling communication. For this reason, we assume here that the number of involved DSO nodes $\left(D_{n}\right)$ would be determined as a function of the supported CSs, i.e., $D_{n}=\max \left(1,\left\lceil\frac{S}{a}\right\rceil\right)$ where $a$ is an aggregation factor, i.e., the number of CSs served by each DSO node. We determine the number of ICN nodes in a similar manner, i.e., $I=\max \left(1,\left\lceil\frac{S}{a}\right\rceil\right)$. That is, the number of both DSO and ICN hosts/nodes increases to cope with the increase of the overall system scale. However, in the ICN scenario, we set $D_{n}=D$ since in this case the scalability of the system relies on the ICN infrastructure.

It can be easily shown that the number of communication sessions in the case of the host-centric paradigm is equal to $D_{n}(P+S)+(E \times O)+S$ while in the case of ICN, it is equal to $S+I\left(E+P+D_{n}+O\right)$. For an example scenario of $E=4, P=100, D=1, O=5, S=1000$ and $a=100$, the host-centric scenario would necessitate the establishment of 12,020 communication sessions against 2,100 in the case of ICN, i.e., a reduction by approximately $82 \%$. Figure 3 , shows how the number of communication sessions scales with the increase of $S$. As the penetration of EVs increases, it is expected that EMSPs/CSOs will deploy numerous CSs to satisfy the increasing demand. Projections from a DSO in the Netherlands even foresee wide scale deployments of several tens of thousands of CSs across the country. We see that ICN achieves a substantial reduction of the number of communication sessions, at the expense of at most 10 ICN nodes in the case of $S=1,000$.

\section{CONCLUSIONS}

In this paper, we took a fresh look on ICN in the context of a new application environment, i.e., smart charging of electric vehicles. Based on a thorough analysis of the application environment currently forming in the Netherlands, we show how ICN can substantially improve the quality and security of the considered application, compared to the currently adopted host-centric model. We show that the emergence of a series 


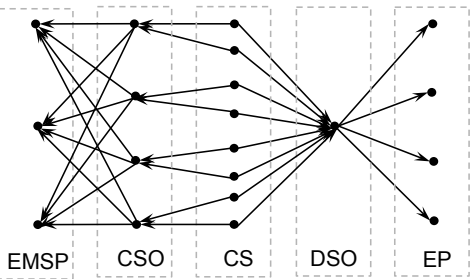

(a) IP

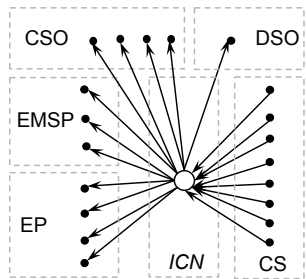

(b) ICN
Fig. 2. Reducing communication complexity. In this example ICN reduces the number of established communication sessions from 32 (in the case of a plain IP), to 20 ( $E=3, P=4, D_{n}=1, O=4$ and $\left.S=8\right)$.

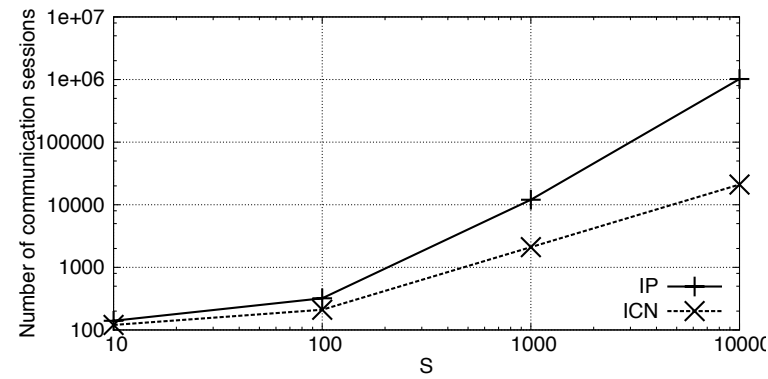

Fig. 3. Number of communication sessions for conveying energy consumption information as a function of the number of the deployed CSs (S). Both axes are in logscale.

of actors in this application environment, along with the multitude of pairwise information flows, result in an unnecessarily complicated communication environment. Based on this observation, we illustrate how ICN can substantially simplify communications through the spatiotemporal decoupling of end-hosts and the inherent ability to support in-network caching and processing. Our preliminary analysis shows that ICN can significantly reduce the number of required communication sessions, requiring the deployment of a limited number of ICN nodes. A more detailed analysis of the potential performance benefits based on real topologies from a DSO in the Netherlands is included in our plans for future work.

\section{ACKNOWLEDGMENTS}

The research leading to these results has received funding from the European Community's Seventh Framework Programme FP7-ICT-2011-8 under grant agreement no. 318708 (C-DAX). The authors alone are responsible for the content of this paper.

\section{REFERENCES}

[1] G. Xylomenos, C. N. Ververidis, V. A. Siris, N. Fotiou, C. Tsilopoulos, X. Vasilakos, K. V. Katsaros, and G. C. Polyzos, "A Survey of Information-Centric Networking Research," Communications Surveys Tutorials, IEEE, vol. 16, no. 2, pp. 1024-1049, Second 2014.

[2] S. Androutsellis-Theotokis and D. Spinellis, "A Survey of Peer-to-peer Content Distribution Technologies," ACM Comput. Surv., vol. 36, no. 4, pp. 335-371, Dec. 2004.
[3] M. Pathan and R. Buyya, "A Taxonomy of CDNs," in Content Delivery Networks, ser. Lecture Notes Electrical Engineering, R. Buyya, M. Pathan, and A. Vakali, Eds. Springer Berlin Heidelberg, 2008, vol. 9, pp. 33-77.

[4] Y. Yan, Y. Qian, H. Sharif, and D. Tipper, "A Survey on Smart Grid Communication Infrastructures: Motivations, Requirements and Challenges," Communications Surveys Tutorials, IEEE, vol. 15, no. 1 pp. 5-20, First 2013.

[5] Y.-J. Kim, M. Thottan, V. Kolesnikov, and W. Lee, "A secure decentralized data-centric information infrastructure for smart grid," Соттиnications Magazine, IEEE, vol. 48, no. 11, pp. 58-65, November 2010.

[6] J. Zhang, Q. Li, and E. Schooler, "iHEMS: An information-centric approach to secure home energy management," in Smart Grid Coтmunications (SmartGridComm), 2012 IEEE Third International Conference on, Nov 2012, pp. 217-222.

[7] K. V. Katsaros, W. K. Chai, N. Wang, G. Pavlou, H. Bontius, and M. Paolone, "Information-centric Networking for Machine-to-Machine Data Delivery - A Case Study in Smart Grid Applications," IEEE Network Magazine, to appear 2014.

[8] C. Bodet, A. Schulke, K. Erickson, and R. Jablonowski, "Optimization of charging infrastructure usage under varying traffic and capacity conditions," in Smart Grid Communications (SmartGridComm), 2012 IEEE Third International Conference on, Nov 2012, pp. 424-429.

[9] E. Rigas, S. Ramchurn, N. Bassiliades, and G. Koutitas, "Congestion management for urban EV charging systems," in Smart Grid Communications (SmartGridComm), 2013 IEEE International Conference on, Oct 2013, pp. 121-126.

[10] K. Mets, F. De Turck, and C. Develder, "Distributed smart charging of electric vehicles for balancing wind energy," in Smart Grid Communications (SmartGridComm), 2012 IEEE Third International Conference on, Nov 2012, pp. 133-138.

[11] K. Dyke, N. Schofield, and M. Barnes, "The Impact of Transport Electrification on Electrical Networks," Industrial Electronics, IEEE Transactions on, vol. 57, no. 12, pp. 3917-3926, Dec 2010.

[12] K. Kok, B. Roossien, P. MacDougall, O. van Pruissen, G. Venekamp, R. Kamphuis, J. Laarakkers, and C. Warmer, "Dynamic pricing by scalable energy management systems - Field experiences and simulation results using PowerMatcher," in Power and Energy Society General Meeting, 2012 IEEE, July 2012, pp. 1-8.

[13] W. Su, H. Eichi, W. Zeng, and M.-Y. Chow, "A Survey on the Electrification of Transportation in a Smart Grid Environment," Industrial Informatics, IEEE Transactions on, vol. 8, no. 1, pp. 1-10, Feb 2012.

[14] PURSUIT Project, PURSUIT Project Home Page, http://www.fp7pursuit.eu, 2011.

[15] V. Jacobson, D. K. Smetters, J. D. Thornton, M. F. Plass, N. H. Briggs, and R. L. Braynard, "Networking named content," in Proc. of the 2009 ACM CoNEXT. New York, NY, USA: ACM, 2009, pp. 1-12.

[16] P. T. Eugster, P. A. Felber, R. Guerraoui, and A.-M. Kermarrec, "The many faces of publish/subscribe," ACM Computing Surveys, vol. 35, no. 2, pp. 114-131, 2003.

[17] I. Psaras, L. Saino, M. Arumaithurai, K. Ramakrishnan, and G. Pavlou, "Name-Based Replication Priorities in Disaster Cases," in Proceedings of the 2nd IEEE workshop on Name-Oriented Mobility, ser. NOM'14. IEEE, 2014.

[18] W. K. Chai, D. He, I. Psaras, and G. Pavlou, "Cache "less for more" in information-centric networks (extended version)," Computer Coтmunications, vol. 36, no. 7, pp. $758-770,2013$.

[19] K. Kursawe, G. Danezis, and M. Kohlweiss, "Privacy-friendly aggregation for the smart-grid," in Proceedings of the 11th international conference on Privacy enhancing technologies, ser. PETS'11. Berlin, Heidelberg: Springer-Verlag, 2011, pp. 175-191.

[20] F. D. Garcia and B. Jacobs, "Privacy-Friendly Energy-Metering via Homomorphic Encryption," in STM, ser. Lecture Notes in Computer Science, J. Cuéllar, J. Lopez, G. Barthe, and A. Pretschner, Eds., vol. 6710. Springer, 2010, pp. 226-238.

[21] D. Boneh, G. D. Crescenzo, R. Ostrovsky, and G. Persiano, "Public Key Encryption with Keyword Search," in EUROCRYPT, C. Cachin and J. Camenisch, Eds., 2004, pp. 506-522. 